\title{
A CASE OF RHINO-ORBITAL MUCORMYCOSIS IN A PATIENT WITH UNCONTROLLED TYPE 2 DIABETES MELLITUS
}

Vikas L1, Ranjith V², Riyaz Ahmed ${ }^{3}$, Surakshith T.K ${ }^{4}$

\section{HOW TO CITE THIS ARTICLE:}

Vikas L, Ranjith V, Riyaz Ahmed, Surakshith TK. "A case of rhino-orbital mucormycosis in a patient with uncontrolled type 2 diabetes mellitus". Journal of Evolution of Medical and Dental Sciences 2013; Vol2, Issue 48, December 02; Page: 9329-9334.

ABSTRACT: Rhinocerebral mucormycosis is a rare life-threatening infection caused by fungi from the order Mucorales. Because of its rapid progression and high mortality, early recognition and aggressive treatment offers the only chance to increase the survival rate. A case of rhinocerebral mucormycosis in a diabetic male patient typically presenting as fever, left periorbital pain and nasal eschar is reported. The clinical manifestations, diagnosis, and treatment of rhinocerebral mucormycosis are discussed. ( J Intern Med Taiwan 2002;13: 160-164 )

KEY WORDS: Rhinocerebral mucormycosis, Diabetic mellitus, immunocompromised state.

INTRODUCTION: Rhinocerebral mucormycosis is a rare life-threatening infection caused primarily by fungi from the order Mucorales. This disease is mostly encountered among immunologically incompetent patients. This acute fulminant fungal infection spreads promptly from the paranasal sinus and orbital regions to the brain in a few days. The mortality rate in patients with systemic mucormycosis is as high as $50 \%$, while in patients with cerebral involvement, it exceeds $80 \% 1$. Because of its rapid progression and high mortality, early recognition and aggressive treatment offers the only chance to increase the survival rate. We reported a diabetic male patient who presented with early typical symptoms. The clinical manifestations,diagnosis and results of treatment are discussed and the literature regarding rhinocerebral mucormycosis is reviewed.

CASE REPORT: A 60 year old male patient who is known hypertensive and diabetic on regular treatment presented to our outpatient department with 5 days history of persistent unilateral (right sided) head ache , fever, retro orbital pain and swelling of the right eye, painful eye movements and diplopia in right lateral gaze.

On examination patient was febrile. Pulse rate was normal and blood pressure was 180/100 $\mathrm{mm}$ Hg. Patient had mild axial proptosis of the right eye and right lateral rectus palsy. Fundoscopic examination of the right eye revealed combined CRAO and CRVO and left eye revealed NPDR with grade III hypertensive retinopathy. Other systems were normal.

A differential diagnosis of right side orbital cellulitis / Tolosa hunt syndrome was made and Patient was admitted at our hospital and routine investigations were ordered along with CT imaging of brain and orbit. Patient was a started on empirical antibiotics and routine investigations revealed uncontrolled diabetes mellitus with chronic kidney disease (stage IV). 


\section{CASE REPORT}

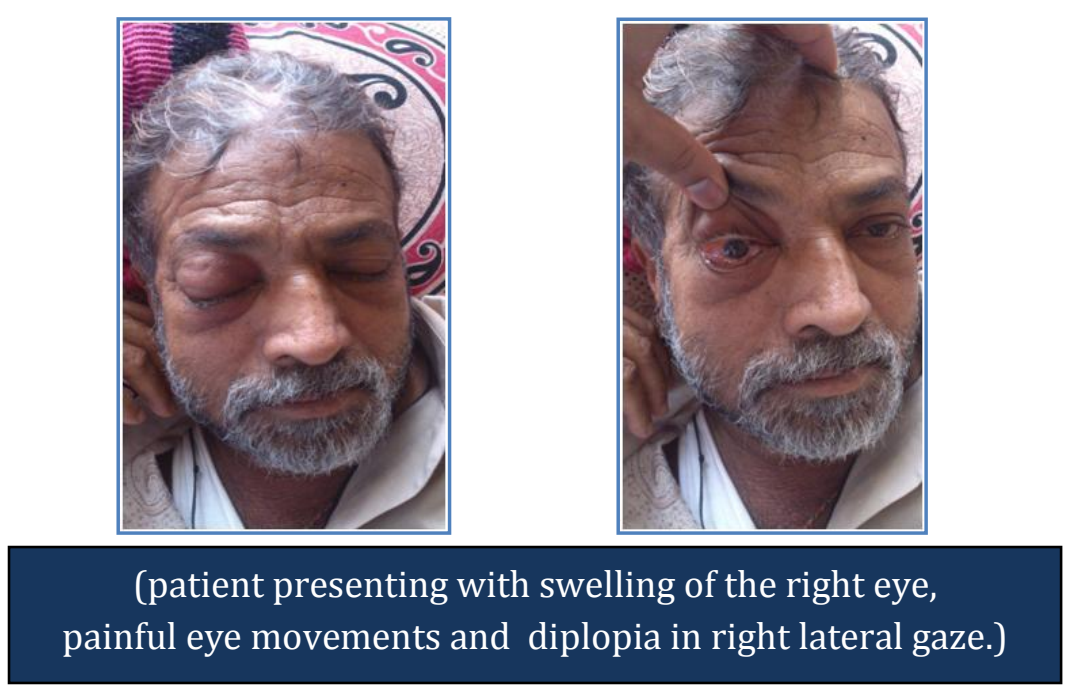

There was no improvement in the clinical course of the patient inspite of the antibiotic theraphy and symptoms worsened. On day three of admission, on examination right eye was frozen (all movements were restricted) axial proptosis increased and complete ptosis with conjunctival congestion was noted. Cornea was clear but sensations were reduced and the direct light reflex was absent and consensual reflex was present. Patient complained of blindness and visual acuity was reduced significantly to just light perception at 1 meter.

Subsequently MRI orbit was ordered and otorhinolaryngologist's opinion was taken and nasal endoscopy revealed fungal debris in the right nostril and biopsy was taken from the ethmoidal sinus and was sent for histopathological examination. MRI showed right sided orbital cellulitis with small abscess in the medial extra conal space with right maxillary, ethmoid and sphenoid sinusitis. Histopathological examination revealed features suggestive of mucormycosis.
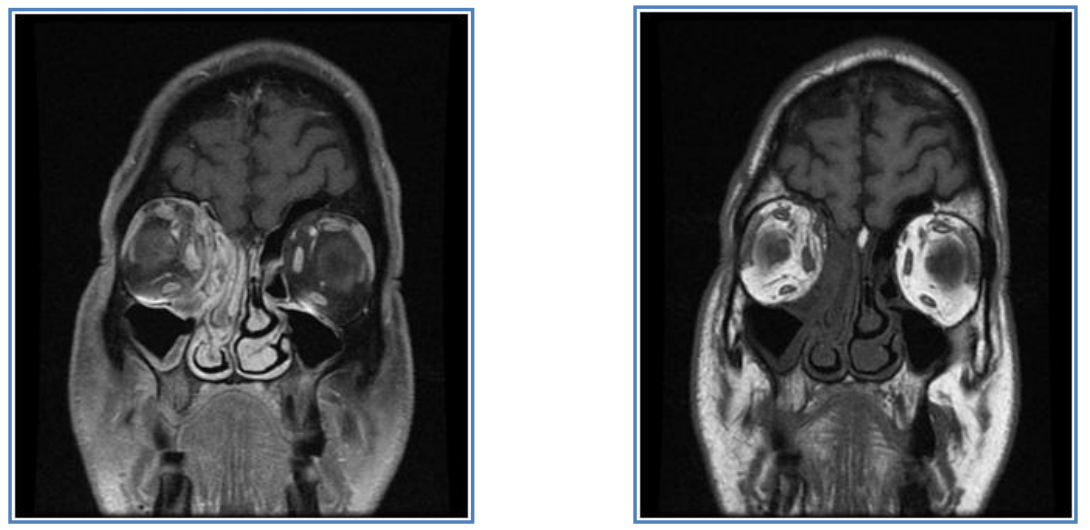

(MRI showing right sided orbital cellulitis with small abscess in the medial extra conal space with right maxillary, ethmoid and sphenoid sinusitis.) 

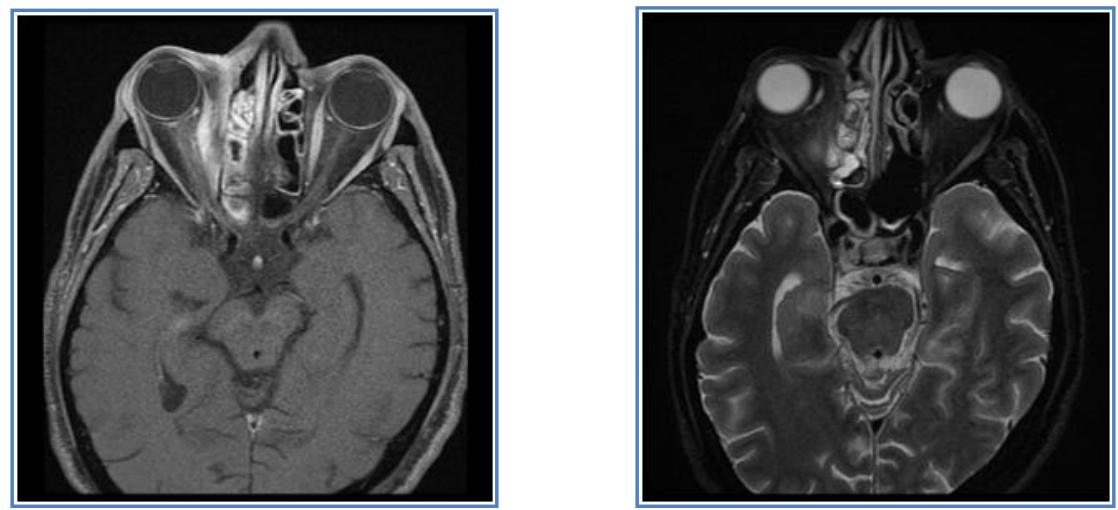

(MRI showing right sided orbital cellulitis with small abscess in the medial extra conal space with right maxillary, ethmoid and sphenoid sinusitis.)

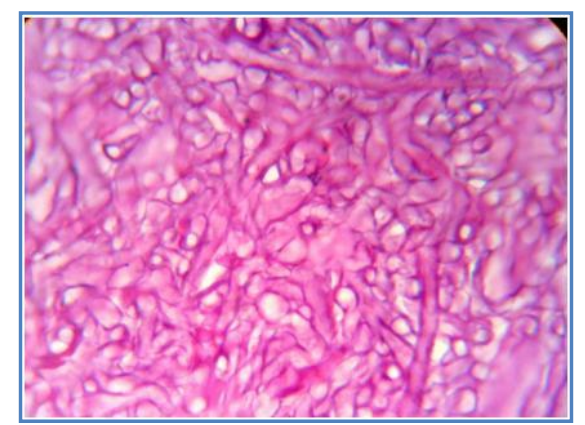

(high resolution microscopic picture of biopsy specimen)

Patient was started on amphotericin B with appropriate dose adjustment based on creatinine clearance (modified eGFR $17.3 \mathrm{ml} / \mathrm{min} / 1.73 \mathrm{~m}^{2}$ ) and patient was treated with low dose amphotericin-B for 7 days and meanwhile his blood sugars were controlled and there was significant improvement clinically, orbital pain, swelling and headache decreased although there was only slight improvement in the visual acuity. Patient was treated successfully and was discharged with insulin. CT scan showed right maxillary and ethmoidal sinusitis and bilateral basal ganglia calcification 16/8/2013 .USG abdomen - grade I MRD with cholelithiasis 24/8/2013. MRI done on 21/8/2013 - evidence of right maxillary, ethmoid, and sphenoid sinusitis. Right minimal preseptal edema noted. Right medial rectus and superior oblique muscles are bulky, fluid alteration in the medial extra conal space with minimal peripherally enhancing collection noted. Haziness of the retro orbital fat noted. Final impression - right sided orbital cellulitis with small abscess in the medial extra conal space with right maxillary, ethmoid and sphenoid sinusitis. Chest X Ray 16/8/2013 fibrotic band in the right upper zone. B.Urea $68 \mathrm{mg} / \mathrm{dL}$. S.Creatinine 3.8mg/dL. eGFR $17.3 \mathrm{ml} / \mathrm{min} / 1.73 \mathrm{~m}^{2}$. 
DISCUSSION: Mucormycosis is a fungal infection caused by a member of the family Mucoraceae. Rhizopus, Mucor, Absidia are the most common isolated from patients with mucormycosis. Rhizopus is responsible for $60 \%$ of all cases of mucormycosis, and $90 \%$ of rhinocerebral mucormycosis 2 . The fungi are found in soil, bread, moulds, rotten fruits and vegetables. It is reported that there are six major clinical symptoms of mucormycosis, presenting as rhinocerebral, pulmonary, gastrointestinal, disseminated, cutaneous and miscellaneous 3.The most common and fatal is rhinocerebral involvement. Though mucormycosis is ubiquitous and grows rapidly, it seldom strikes in immunologically competent patients. Therefore, if an infection with mucormycosis occurs, it usually indicates a serious underlying medical condition. The risk factors for developing rhinocerebral mucormycosis include severe burn, acquired immune deficiency syndrome (AIDS), immunosuppressive medications, leukemia, diabetes mellitus, and organ transplantation 4. About $70 \%$ to $80 \%$ of these patients have diabetes mellitus. As is reported in our case, most diabetics who develop rhinocerebral mucormycosis (RCM) are in poor metabolic control with complicating ketoacidosis 5. It is suggested that fungal organism grows in ketotic patients because acidosis disrupts iron binding to transferrin and the result increases in free iron then promotes growth of the fungus. At the same time, high blood sugar level may also alter the immunologic capability to resist mucormycosis through reduction of WBC chemotaxis and the ability of macrophages 6 .

As seen in our case, diabetic patients with poor controlled blood sugar who develop rhinocerebral mucormycosis typically present with malaise, retro-orbital headache, fever, and occasionally dark, blood-tinged rhinorrhea. Originally the oral and nasal cavities may reveal a black eschar on the plate, septum or turbinate that may then involve the orbit via the communicating foramina and venous channel 7. Unilateral ptosis and pupillary dilatation imply involvement of cranial nerve V and VII. Because the disease provokes diffuse tissue necrosis, the fungi can easily invade the wall of blood vessels, leading to thrombosis and tissue ischemia 8. Therefore, it is not uncommon to find the infection spreading to the cavernous sinus or the central nervous system. The deterioration in mental status is an ominous sign, often heralding intracerebral extension of the disease process. All of these symptoms may develop over a period of several days or may occur as a fulminating process within hours 2. Imaging studies are important to evaluate the extent of the disease. CT of patients with rhinocerebral mucormycosis shows opacification of the paranasal sinus and thickening of the sinus mucosa and bone destruction without an air-fluid level. In addition, when the orbit is invaded, increased density of the orbital fat and venous engorgement may be seen 9 . Magnetic resonance imaging (MRI) can demonstrate soft tissue lesions better, especially in diagnosis of cavernous sinus thrombosis.

Biopsy of the affected tissue is required to confirm the infection. On histologic section, these organisms are characterized by wide, non-septate hyphae with right-angled branching 10. Cultures are still the standard means of diagnosis. But even positive histologic findings, routine sinus cultures and blood cultures are rarely positive.

Treatment of rhinocerebral mucormycosis should consist of prompt control of hyperglycemia and ketoacidosis, aggressive surgical debridement of involved tissue, and administration of parenteral amphotericin B. Amphotericin B has potential renal toxicity and its dosage should be individually adjusted between $0.5 \mathrm{mg} / \mathrm{Kg} /$ day and $1.0 \mathrm{mg} / \mathrm{Kg} /$ day, based on the body weight and renal function of the patients 11. A total cumulative dosage of 2 to $4 \mathrm{~g}$ is generally advocated. 


\section{CASE REPORT}

Hyperbaric oxygen (HBO) treatments should also be considered for those patients with aggressive infections. A few studies have shown that HBO has direct in-vitro fungistatic activity and reduce tissue hypoxia, which may reverse the hypoxic acidosis that helps the fungi to proliferate 12 . Blitzer and Lawson found that in their review of 170 cases of RCM, $63 \%$ of untreated diabetics died as compared with $17 \%$ mortality rate when therapy included aggressive surgery and amphotericin B administration 5. The importance of surgery is pronounced when no surgical treatment or only biopsy was performed the mortality rate is as high as $58 \%$.

CONCLUSION: Rhinocerebral mucormycosis is an acute opportunistic mycosis that predominantly occurs in the patients with diabetes. The clinic physician may see patients with RCM in its earliest stages masquerading as other less serious diseases. Early diagnosis, aggressive surgical debridment, high dose amphotericin B and good control of blood sugar are the most important factors to decrease the morbidity and mortality from this fungal disease.

\section{REFERENCES:}

1. Lantz NJ, Golis TA. Sphenoidal mucocele with cavernous sinus invasion. J Am Optom Assoc 199364 583-585.

2. McCarthy WW, Frenkel M, Busse BJ. Visual loss as the only symptom of sphenoid sinus mucocele. Am J Ophthalmol 197274 1134-1140.

3. Nithyanandam S, Jacob MS, Battu RR. Rhino-orbital-cerebral mucormycosis: a retrospective analysis of clinical features and treatment outcomes. Indian J Ophthalmol 2003 51 231-236.

4. Damante JH, Fleury RN. Oral and rhinoorbital mucormcosis. J Oral Maxillofac Surg 199856 267-271.

5. Paulltauf A. mycosis mucorina. Virchows Arch 1885102 43, cited by Brian M, O’Neill DDS, Alessi AS, George EB and Piro J. Disseminated rhinocerebral mucormycosis. J Oral Maxillofac Surg 200664 326-333.

6. O'Neill BM, Alessi AS, George EB and Piro J. Disseminated rhinocerebral mucormycosis. J Oral Maxillofac Surg 200664 326-333.

7. Schwartz JN, Donnelly EH, Klintworth GK. Ocular and orbital phycomycosis. Surv Ophthalmol 197722 3-28.

8. Spellberg B, Edward J, Abrahim A. Novel perspective on mucormycosis: pathophysiology, presentation and management. Clin Microbiol Rev 2005 18556-569.

9. McNab AA, McKelvie P. Iron overload is a risk factor for zygomycosis. Arch Ophthalmol 1997 115 919-921.

10. Van Cutsem J, Boelaert JR. Effects of deferroxamine, ferroxamine and iron on experimental mucormycosis.

11. Garrison MW, Campbell RK. Identifying and treating common and uncommon infections in the patient with diabetes. Diabetes Educ 1993 19 522-529.

12. Dokmetas HS, Canbay E, Yilmaz S, Elaldi N, Topalkara A, Öztoprak İ and Yildiz E. Diabetes ketoacidosis and rhino-orbital mucormycosis. Diabetes Res Clin Pract 200257 139-142.

13. Lehrer RI, Howard DH, Sypherd PS: Lehrer RI. Mucormycosis. Ann Intern Med 1980 93 93-108. Rocha G, Garza G, Font RL. Orbital pathology associated with diabetes mellitus. Int Ophthalmol. 


\section{AUTHORS:}

1. Vikas L.

2. Ranjith V.

3. Riyaz Ahmed

4. Surakshith T.K.

\section{PARTICULARS OF CONTRIBUTORS:}

1. Assistant Professor, Department of Medicine, Mysore Medical College \& Research Institute, Mysore.

2. Senior Resident, Department of Medicine, Mysore Medical College \& Research Institute, Mysore.

3. Senior Resident, Department of Medicine, Mysore Medical College \& Research Institute, Mysore.
4. Senior Resident, Department of Medicine, Mysore Medical College \& Research Institute, Mysore.

\section{NAME ADRRESS EMAIL ID OF THE CORRESPONDING AUTHOR:}

Dr. Vikas L, $\# 11,9^{\text {th }}$ Main, $6^{\text {th }}$ Cross, Brindavan $1^{\text {st }}$ Stage, Mysore -570020 .

Email - vikilaxman@gmail.com

Date of Submission: 17/10/2013.

Date of Peer Review: 18/10/2013.

Date of Acceptance: 20/11/2013.

Date of Publishing: 26/11/2013 